\title{
Maintenance Orientation and Practices in Colleges of Education in Ghana: A Stakeholder Perspective
}

\author{
Perpetua Emma Gyenfie \\ Christina Bukari \\ Department of Technical Education \\ Mampong Technical College of Education \\ P.O. Box 31, Mampong Ashanti. \\ Michael Tsorgali \\ Department of Technical Education \\ University of Education Winneba-Kumasi \\ P.O. Box 1277 \\ Kumasi. \\ Mary Opokua Ansong \\ Henry Asante Antwi \\ Department of Computer Science \\ Faculty of Applied Sciences and Technology \\ P.O Box 854, Kumasi.
}

\begin{abstract}
This study has explored issue relating to orientations and practices relating to maintenance schedule, maintenance inspections, maintenance planning, maintenance reporting, maintenance process based on information collected from selected stakeholders in the operations of Colleges of Education in Ghana. Data was collected from teachers and students in Colleges of Education in Ghana. Generally the analysis suggests that maintenance practices and orientation, both among students and staff is relatively low. A direct and related consequence of this problem is the delays in the execution of maintenance works. Also, the Colleges of Education do not have a fully equipped maintenance unit as well as qualified personnel. The implication is that the few tradesmen do the minor maintenance works sometimes with improvised equipment. It is recommended that the process through which maintenance requests and budget proposals go through should be revisited and reduced to the barest minimum in order to facilitate quick reactions to complaints when they are reported. Secondly, maintenance Clubs should be set up in the Colleges to do periodic inspection of buildings that is a post occupancy evaluation every quarterly. This will enable the maintenance needs of the buildings to be discovered in time before the situation gets out of hand. Officers should oversee and ensure that thorough periodic inspections of buildings' conditions are done. Further, building inspection should be planned, because proper planning is a sure way to lessen cost of maintenance since doing so can provide perception into future maintenance needs and avoid unnecessary costs.
\end{abstract}

\section{Introduction}

Maintenance culture has been recognized as an important aspect to increase the quality of maintenance work. Despite being unique to each organization, culture within organizations needs to evolve to suit the ever changing market demands and trends. The cultivation of maintenance culture should commence with the change of mindsets and attitudes to promote continuous knowledge and skill enhancement, and performance improvement in maintenance activities . Cultural change needs to break indigenous moulds of poor perception, old patterns of now inappropriate behaviour, and outdated beliefs and values. Though studies pertaining to culture are abundant in business and manufacturing sectors, little effort has been put to study culture in the construction industry, particularly in maintenance. Maintenance work has not been studied much from the cultural perspective and former approaches have seldom taken into account the demands of the maintenance work in the entire organization Reiman, T. and Oedewald, P. (2004). Maintenance culture is important to elevate maintenance performance which would directly lead to enhanced facilities performance (Haroun \& Duffuaa, 2009). It is an alternative for improving maintenance commitment and creating maintenance awareness among all parties in maintenance management. 
In Ghana, there are claims that maintenance culture which encompasses provision for adequate care of the hard earned infrastructure has not gained ground in the consciousness of resource managers over the years (especially in educational sector) and consequently, several government buildings at the verge of collapsing. In Ghana evidence of abandoned factory plants and machinery, dilapidated school buildings, pot-holes and chasms on the constructed highway roads, deserted vehicles with minor problems, moribund industries and a host of other property belonging to the country which have little or insignificant problem. These actions in myriad ways have resulted in a colossal waste of scarce resources hence a major problem that continues to ravage and undermine institutional sustainability in developing countries.

In the educational sector in Ghana, concerns about maintenance culture have been raised at the governmental, ministerial, and administrative levels. For example a former Minister of Agriculture, recently indicated at a speech and prize giving day celebration of the St. Augustine's College in Cape Coast that the lack of maintenance culture in Ghana was causing the nation a great deal of money which could have been channeled in to development projects. He stressed the fact that the attitude of Ghanaians is to "build, neglect and rehabilitate" instead of going by the axiom that "a stitch in time saves nine" recorded by GNA, 2012. However, even those in government themselves have been accused of similar lackadaisical attitude towards the maintenance of public properties over which they superintend. Following their recent upgrade to tertiary institutions, it has been the estimation of educational reform that facilities in these institutions will be upgraded to meet the standard of academic and para-academic responsibilities undertaken in these institutions as well as the new demands of tertiary education. However, reports from several studies on the infrastructural situation in Ghana's 38 public Colleges of Education show a consistent decline in the quality of infrastructure due largely to lack of maintenance practices. To proffer effective solutions to the challenge, an understanding of the maintenance orientation and practices in these institutions is essential; a concern which this study is devoted to. The study explores issue orientations and practices relating to maintenance orientation, maintenance schedule, maintenance inspections, maintenance planning, maintenance reporting, maintenance process based on information collected from selected stakeholders in the operations of Colleges of Education in Ghana.

\section{Related Works}

\section{Maintenance Policy}

Maintenance policy has been defined as a strategy within which decisions on maintenance are taken. It may alternatively be defined as the basic rules for the allocation of resources (men, materials and money) between the alternative types of maintenance actions that are available and necessary to management. In order to make a rational allocation of resources, the benefits of those actions to the organization as a whole must be identified and related to the costs involved. Maintenance policy should be considered in the widest possible context throughout all the phases in the life cycle of a building. Furthermore, it needs to be recognized that policy influences on maintenance may not always be direct ones. In other words, it is possible to distinguish clearly between policy that is specifically directed toward building maintenance and policy decisions taken with respect to other matters, but which will influence maintenance.

The attitude of, or stance taken by, decision makers during the procurement of a building will have a profound influence, not only on the strategic design but also on the building's subsequent performance. All decisions should be carefully examined, and the possible consequences for the building throughout its economic life considered. In simple terms, the building cycle can be described in the following six stages: brief, design, procurement, construction, commissioning, and operation (Campbell, 2001). The decisions taken relating to all these stage will have a bearing on the maintenance requirements of the building. Whatever scenario exists, in considering the operation of maintenance management there are a number of common areas requiring a policy statement include Resource Allocation. The proportion of resources that will be allocated to building maintenance will have to be determined in a competitive environment. These resources may be in terms of finance, staffing (both managerial and operative) and time. Generally, maintenance tends to compete on rather unfavorable terms for all of these, and for finance in particular. Following the allocation of maintenance resources as a block is the need to decide precisely how these resources are to be distributed. Given the inevitable pressures, this may be carried out in a variety of ways, some of which may have little to do with building performance considerations and be beyond the influence of the maintenance technical staff. The process may be the result of a clearly defined policy or of some mysterious internal process dictated by other characteristics of the organisation. Here too we need to recognize that the resource allocation may be part of an outsourced operation that will have contractual implications to be considered and may be heavily influenced by a bidding process subjected to a range of market pressures. 


\section{Maintenance Orientation and Practices}

Maintenance is a generic term which includes planned maintenance, repair and reimbursement and provision of replacement of the structure. The goal of maintenance is to sustain the life of major assets whether building or infrastructure co-operation under the auspices of United Nations Educational, Scientific and Cultural Organization (UNESCO). British standard (BS 583) (2004) define maintenance as the combination of all technical and associated administrative actions intended to retain an item in, or restore it to, a state in which it can perform its required function. The requirement for maintenance must not be less than those necessary to meet the relevant requirements and 'maintained' is defined in factories act 1961 as "maintained in an efficient state, in efficient working order and in good repair" According to British Standard (BS 3811) maintenance is defined as "works undertaken in order to keep or restore every facility i.e. every part of a building, site and concept to an acceptable standard". An acceptable standard in building terms is one which sustains the utility and value of the facility.

In general, the goal of any organization is to increase profitability by providing quality products, prompt or complete service as well as swift delivery. In today's competitive world, being successful often entail how well a company is able to make change and adapt to those changes swiftly. This can be seen as a way or technique of dealing with outfashioned or out-of-date managerial approach and other forms of managerial practices. According to Wireman (2000) Maintenance as a support function together with asset management can be a driving force in this regards in two major ways, i.e. by decreasing the cost of running buildings and machine while also increasing capability. Hence, with the growth of mechanisation and automation, which mean that a component could fail at any given time leading to interrupted operations, availability and reliability becomes a keyword. Al-Najjar (2007) expressed that institutions should be able to utilize its valuable and rare resources efficiently and effectively to attain the long-term high profitability, regardless of which prospective, outside-in (i.e. external factors, such as emerging of institutions, partnership and market structure) and inside-out (i.e. internal factors, such as institution's resources, competence and differentiation) that is adopted or implemented by the institution's management. In addition, the negligence of maintenance and its role in the education processes allows swift degradation of structures and its resultant service quality.

Maintenance organization according to Haroun \& Duffuaa (2009) may take an immeasurable number of forms, the best for a particular situation being determined by systematic consideration of factors as maintenance work load and its pattern, cost of unavailability, location of plant, amount of emergency work, production organization and maintenance resources. Haroun \& Duffuaa,(2009) further stated that in many cases the problem is one of achieving the optimum balance between plant availability and maintenance resource utilization. The dominant factor in the design of a maintenance organization is unavailability cost. Maintenance organization can be considered as being made up of three essential and interconnected components. They are as follows:

- Resources-personnel, spare parts and tools of a particular size, composition, location and movement.

- Administration-a hierarchy of authority and responsibility for deciding what, when, and how work should be carried out.

- Work planning and control system-a mechanism for planning and scheduling the work and feeding back information which is needed if the maintenance effort is to be correctly directed towards its defined objective.

\section{Quality and Maintenance Management}

Quality is becoming a business strategy leading to success, growth and enhanced competitive position; so organisations strive to attain that position through maintaining the asset in ideal operating conditions. Hence, one can see that quality, as a general concept is a demand for all sides of life; it has a strong link to the humanity improvement and style. It seems to be the golden thread, which motivates anyone to catch it. Quality can fit in anywhere and at any time under certain conditions such as the availability of resources accompanied with full commitment of all interested people. Therefore, despite its difficulty to achieve, quality depends on the willingness and interest of the people in any place and at any time. Quality in general could be expressed in the single words such as best, perfect, optimal and excellence. The link between maintenance and quality, although not completely missing, is not adequately addressed in the literature. Although the importance of keeping all parts of any asset in proper working condition cannot be overemphasized, so many organisations do not pay enough attention to maintenance as integrated activities. Increasing the capacity of products and providing goods and services can be gained by maintaining the physical assets with crucial care. It also can expand the ability to produce goods and services to the customer's satisfaction consistently. Furthermore, it could predictably provide a safe and controlled work or service environment with a minimum of risk. However, keeping an organization working effectively is a complex job. There are several key concerns in forms of questions which impact the cost of asset maintenance consistent with Campbell's (2005) perspective as follows;

- How does an organisation attract and keep capable people to maintain huge asset? 
- What is the optimum level of inventory of maintenance parts, materials, and consumables, and what organisational arrangements are appropriate?

- Does an organisation need specialist maintenance engineering support?

Although these questions are not new, nowadays, global competitive stakes make it more important due to the concept of "maximise output of goods and services and minimise input of resources-financial, human, and physical". However, providing the best value is environmentally conscious and has to do with giving the best quality, at the suitable price. As stated the following equation of value; Value $=($ Quality $*$ Services $) 1($ Cost*Time*Risk). The higher the quality of service for a given cost and response time, the more value it will be to the customer. Therefore, the physical resources employed must be available when needed, and must produce at the required rate and quality, all at reasonable cost. Environmental and safety risks must be minimised. Schuman \& Brent (2005) opined that, there are some innovative approaches in maintenance management; many organisations are working to gain competitive edge through implementing it such as; preventive maintenance PM, total productive maintenance TPM, reliability centred maintenance RCM, tero-technology, Computerised Maintenance Management Systems CMMS, and condition-based maintenance CBM (Wienker, M., Henderson K., and Volkerts J. (2016).

\section{Elements of a Maintenance System}

The maintenance system comprises both formal and informal elements. The formal elements include management systems and methods, organisational structures, information systems, and technology necessary to implement the generic maintenance tasks within an industrial enterprise. The management system essentially specifies when to deal with one or more of the maintenance tasks, what to do when the task is dealt with, and who should do it. The management system also includes the formal collective knowledge and know-how. Regarding the organisation in some industrial firms, there is a distinct division of responsibility for maintenance tasks between operators of individual machining tools, set-up fitters and skilled workers from the maintenance department An information system can range from manual data collection and reporting to sophisticated systems .

Informal elements of a maintenance system include the actors of maintenance. These actors include the individuals performing maintenance functions (i. e. technicians, operators, managers) as well as the influence of the corporate culture. Individuals of a maintenance organisation act and decide using their knowledge, skills and know-how, their motivation and attitudes. In addition to being part of the formal management system, individuals act informally. Attention is given to how individuals from the maintenance department interact with other members of the organisation. One of the design variables for improving maintenance with respect to individuals is to educate them by providing appropriate decision support in terms of information systems and decision rules. Although there are only a few possibilities to alter directly the culture in an industrial enterprise, it is suggested that corporate culture be included as an element in a maintenance system. It captures the collective, informal learning, which has occurred in the past. So the maintenance system is very much dependent on the structure and kind of firm/institution, the external conditions, internal constraints and specified corporate objectives. This analysis of the situation leads into the identification of the object system or focal areas for maintenance and the maintenance task profile.

\section{Materials and Methods}

Data was collected using quantitative methods. The self-administration method was used for questionnaires designed for the staff and students of selected Colleges of Education located within the 10 regions of Ghana. These were grouped into zones based on the zonal classification of the Ghana Education Service. Predominantly close-ended questions were designed to gather information from the 145 respondents. The questionnaire for the students was made up of twentyseven (27) questions as well as that of the Staff. The questionnaires were pre-tested to assess it reliability and validity for the objectives of the study. The responses from the pre-testing were used to modify items and the necessary corrections were made on the data collection instrument for the main study. Two Field supervisors and five Research Assistants were recruited one from each of the five colleges and trained to work with the Principal Investigator. Data was coded, sorted, analyzed by computer software Statistical Package for Social Sciences (SPSS).

\section{Data Analysis}

The appropriateness of the data for factor analysis was determined using the Kaiser-Meyer-Olkin measure of sampling adequacy (KMO-MSA) and Bartlett's Test of Sphericity. The KMO value recorded was greater than 0.60 and the value of the Bartlett's Test of Sphericity was very significant. Further a Varimax rotation and principle components analysis for factor analysis was performed after which all the factors with a factor loadings lower than 0.50 were eliminated. This was followed by conducting a Cronbach's alpha reliability analysis. 
It was ensured that all measures of sampling adequacy exceeded the Cronbach's alpha reliability value threshold level of 0.60 and large and significant Bartlett's Test of Sphericity. To test the significance of significance of opinion of respondents, one sample $t$ test model was formulated as follows:

$t=\sqrt{p}\left(\frac{x}{s}\right)=\sqrt{p}\left((\bar{X}-\mu) /\left(\frac{\delta}{\sqrt{n}}\right)\right) / s$

Where $\bar{X}$ is the sample mean from the sample $\mathrm{X}_{1}, \mathrm{X}_{2}, \mathrm{X}_{3, \ldots \ldots \ldots,} \mathrm{X}_{\mathrm{n}}$ of size $n, s$ is the ratio of sample standard deviation over population standard deviation, $\sigma$ is the population standard deviation of the data, and $\mu$ is the population mean. The assumptions of the test are:

- X follows a normal distribution with mean $\mu$ and variance $\sigma^{2}$

- $S^{2}$ follows a $X^{2}$ distribution with $p$ degrees of freedom under the null hypothesis, where $p$ is a positive constant

- $Z$ and $s$ are independent.

\section{Results}

ANOVA test Analysis of the Maintenance Orientation and Practices

Table 1. One-Sample Statistics

\begin{tabular}{|l|r|r|r|r|}
\hline & \multicolumn{1}{|c|}{$\mathrm{N}$} & \multicolumn{1}{c|}{ Mean } & Std. Deviation & Std. Error Mean \\
\hline Maintenance Orientation & 247 & 3.7652 & 20.46695 & 1.30228 \\
(Attitude) & 247 & 2.3968 & 1.70737 & .10864 \\
Maintenance Need & 245 & 3.8531 & 2.51773 & .16085 \\
Inspection & 247 & 1.3684 & .68513 & .04359 \\
Maintenance Scheduling & 249 & 3.3735 & 1.20527 & .07638 \\
Maintenance Execution & & & \\
Duration of Maintenance & & & \\
Rectification &
\end{tabular}

Table 2 One-

Sample T -Test

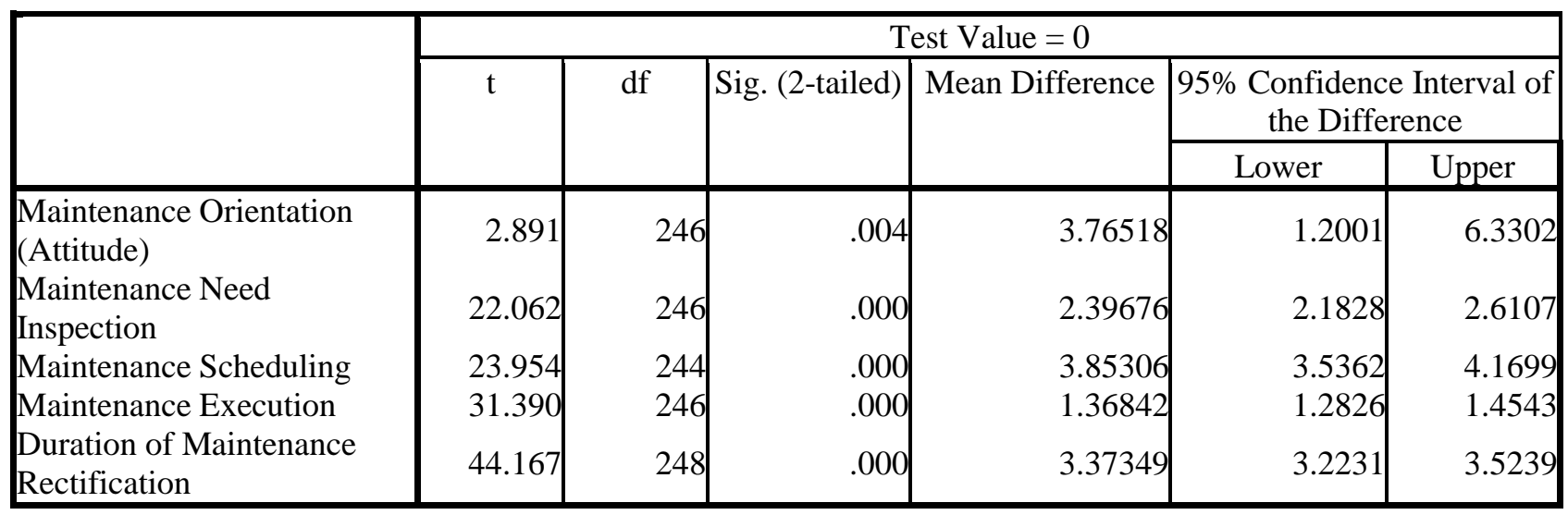

Table 1 and 2 present the descriptive statistics and one sample t-test of responses of teachers and students about maintenance practices and orientation in Colleges of Education. The analysis shows that the respondents generally agreed that little orientation is provided about maintenance schedule in the college for both students and teachers when they come to the school. The mean difference of response is 3.76518 on a five point likert scale and this is statistically significant (Sig. value $=.004<0.05$ ). In the same regard, the mean response value of 2.39676 also indicates that the rate at which maintenance inspection is undertaken in the schools is unsatisfactory and this is also statistically significant at $95 \%$ confidence interval $($ Sig. value $=.000<0.05$ )

When the respondents were asked about the extent to which the maintenance schedule notices are given in the school, the responses were also generally negative as shown in the mean response value and statistical significance level (Sig. value $=.000<0.05)$. The trend analysis showed that staff $(40.0 \%)$ each in both ERGR and ASHBA zones indicated they have maintenance schedule for staff residence while $(25.5 \%)$ of students in ERGR zone indicated they have no maintenance schedule. The result again shows that majority of both staff (88.0\%) and students (63.0\%) responded there is no maintenance schedule notices at the residences of staff and students in general. 
Many works verified that the lack of linkage between policy and the overall corporate strategy of manufacturing/services organisation leads to poor maintenance performance (Jonsson, 2007). Maintenance plans help in scheduling maintenance work and allocating the resources for each task. The lack of planning can significantly restrict a maintenance operation from meeting its objectives in the organisation. Consequently proper planning can lead to high responsiveness, and less unplanned work (Cholasuke, et al. 2004). Regarding the extent to mean response value about the execution of maintenance, the analysis also shows that the respondents think it is not done frequently. This is indicated by the weak mean response value of 1.36842 which is also statistically significant (Sig. value $=.000<0.05$ ). Finally, the duration of maintenance rectification has also been analysed. Staff and students of the colleges were asked to indicate how often school does maintenance on bungalows on a scale of 1 to 5 . The results show that higher percentage (24.7\%) of students in ASHBA indicated the school does maintenance work "within a month" while $40.0 \%$ of staff in the CRWR zone indicated maintenance work on bungalows were done "within a year". The result further shows that majority of staff $(88.0 \%)$ and students $(59.0 \%)$ from all the zones indicated maintenance works are carried out within a month.

\section{Discussion and Conclusion}

This study has explored issue relating to orientations and practices relating to maintenance schedule, maintenance inspections, maintenance planning, maintenance reporting, maintenance process based on information collected from selected stakeholders in the operations of Colleges of Education in Ghana. Generally the analysis suggests that maintenance practices and orientation in Colleges of Education both among students and staff is relatively low. A direct and related consequence of this problem is delay in the execution of maintenance works. Also, the Colleges of Education do not have a fully equipped maintenance unit as well as qualified personnel. The implication is that the few tradesmen do the minor maintenance works sometimes with improvised equipment. As a result bulk maintenance activities that need urgent attention are always deferred especially those on the multy- storey buildings. It has been revealed that the maintenance culture of Colleges of education is relatively healthier as compared to other public institutions. This may be partly attributable to the innovativeness of the management of the Colleges. This is not to say that Colleges of education has not got its own maintenance challenges. The causes of maintenance problems at Colleges of education have been examined with the principal cause being financial problems, the absence of an independent functioning estate unit, deferred maintenance and non-compliance with the terms of the Colleges of Education maintenance policy. It is recommended that the process through which maintenance requests and budget proposals go through should be revisited and reduced to the barest minimum in order to facilitate quick reactions to complaints when they are reported. Secondly, maintenance Clubs should be set up in the Colleges to do periodic inspection of buildings that is a post occupancy evaluation every quarterly. This will enable the maintenance needs of the buildings to be discovered in time before the situation gets out of hand. Officers should oversee and ensure that thorough periodic inspections of buildings' conditions are done. Further, building inspection should be planned, because proper planning is a sure way to lessen cost of maintenance since doing so can provide perception into future maintenance needs and avoid unnecessary costs. Moreover, tenancy agreements should be reviewed to transfer the responsibilities of at least internal repairs to occupants (staff). Revised tenancy agreements if properly enforced would ensure that the maintenance section will only have to be concerned with major and external repairs on the buildings. It must be noted that there should be urgent repair work on the cracked walls, roof system and paintings of the dilapidated buildings to make them appropriate with the status of the Colleges.

Future work

In future we will investigate the use of computerized system to augment or improve maintenance in buildings.

\section{Reference}

Al-Najjar, B. (2007). Condition-based maintenance: Selection and improvement of a cost-effective vibration-based policy in rolling element bearings. Unpublished doctoral dissertation, Lund Institute of Technology, Lund University, Sweden.

British Standard (BS3811:2004) : comprehensive Based of Terminology, Symbols,

Campbell, J.D. \& Jardine, A.K.S. (2001). Maintenance Excellence Optimizing Equipment Life-Cycle Decision: Marcel Dekker, USA.

Cholasuke, C., Bhardwa, R., \& Antony, J. (2004). The status of maintenance management in UK manufacturing organisations: Results from a pilot survey. Journal of Quality in Maintenance Engineering: 10(1), 5-15 
GNA (2012). Lack of maintenance culture causing nation huge sums.

http://ghananewsagency.org/social/lack-of-maintenance-culture-causing-nation-huge-sums-ahwoi-40828

Haroun, A. E., and Duffuaa, S.O. (2009) "Maintenance Organization." In Handbook of Maintenance Management and Engineering, 3-15: Springer.

Jonsson, P. (2007). The Impact of Maintenance on the Production Process-Achieving High Performance: Lund University, Institute of Technology ,Department of Industrial Engineering, Division of Production Management

Schuman, C. A, \& Brent, A. C. (2005). Asset life cycle management: towards improving physical asset performance in the process industry. International Journal of Operations \& Production Management, 25(6), 566-579.

Wireman, T. (2000). Developing performance indicators for managing maintenance. $2^{\text {nd }}$ Edition. New York: Industrial Press.

Wireman, T. (2005). Developing performance indicators for managing maintenance. Industrial Press Inc..

Wienker, M., Henderson K., and Volkerts J. (2016) "The Computerized Maintenance Management System an Essential Tool for World Class Maintenance." Procedia Engineering 138: 413-20

Reiman, T. and Oedewald, P. (2004) "Measuring Maintenance Culture and Maintenance Core Task with CultureQuestionnaire—a Case Study in the Power Industry." Safety science 42, no. 9: 859-89. 